TRANSACTIONS OF THE

AMERICAN MATHEMATICAL SOCIETY

Volume 354, Number 11, Pages 4627-4637

S 0002-9947(02)03074-X

Article electronically published on June 4, 2002

\title{
BROWNIAN-TIME PROCESSES: THE PDE CONNECTION II AND THE CORRESPONDING FEYNMAN-KAC FORMULA
}

\author{
HASSAN ALLOUBA
}

\begin{abstract}
We delve deeper into our study of the connection of Brownian-time processes (BTPs) to fourth-order parabolic PDEs, which we introduced in a recent joint article with W. Zheng. Probabilistically, BTPs and their cousins BTPs with excursions form a unifying class of interesting stochastic processes that includes the celebrated IBM of Burdzy and other new intriguing processes and is also connected to the Markov snake of Le Gall. BTPs also offer a new connection of probability to PDEs that is fundamentally different from the Markovian one. They solve fourth-order PDEs in which the initial function plays an important role in the PDE itself, not only as initial data. We connect two such types of interesting and new PDEs to BTPs. The first is obtained by running the BTP and then integrating along its path, and the second type of PDEs is related to what we call the Feynman-Kac formula for BTPs. A special case of the second type is a step towards a probabilistic solution to linearized Cahn-Hilliard and Kuramoto-Sivashinsky type PDEs, which we tackle in an upcoming paper.
\end{abstract}

\section{IntRodUCTION AND STATEMENTS OF RESULtS}

Let $B(t)$ be a one-dimensional Brownian motion starting at 0 and $X^{x}(t)$ be an independent $\mathbb{R}^{d}$-valued continuous Markov process starting at $x$, both defined on a probability space $\left(\Omega, \mathcal{F},\left\{\mathcal{F}_{t}\right\}, \mathbb{P}\right)$. We call the process $\mathbb{X}_{B}^{x}(t) \triangleq X^{x}(|B(t)|)$ a Brownian-time process (BTP): the regular clock $t$ is replaced with the Brownian clock $|B(t)|$. In the special case where $X^{x}$ is a Brownian motion starting at $x$ we call the process $\mathbb{X}_{B}^{x}(t)$ a Brownian-time Brownian motion (BTBM). Excursions-based Brownian-time processes (EBTPs) are obtained from BTPs by breaking up the path of $|B(t)|$ into excursion intervals - maximal intervals $(r, s)$ of time on which $|B(t)|>0$ - and, on each such interval, we pick an independent copy of the Markov process $X^{x}$ from a finite or an infinite collection. Frequently in applied PDEs (like the Allen-Cahn and Cahn-Hilliard and others), an order parameter $\epsilon$ with some physical significance is an important part of the PDE; and to accommodate such a parameter, we introduce the $\epsilon$-scaled BTPs $\mathbb{X}_{\epsilon B}^{x}(t) \triangleq X^{x}(\epsilon|B(t)|)$ and their excursion cousins (see Theorem 1.2 below for a PDE connection).

Received by the editors November 5, 2001 and, in revised form, April 9, 2002.

2000 Mathematics Subject Classification. Primary 60H30, 60J45, 60J35; Secondary 60J60, $60 \mathrm{~J} 65$.

Key words and phrases. Brownian-time processes, initially perturbed fourth order PDEs, Brownian-time Feynman-Kac formula, iterated Brownian motion.

Supported in part by NSA grant MDA904-02-1-0083. 
BTPs and EBTPs may be regarded as canonical constructions for several famous as well as interesting new processes. To see this, observe that the following processes have the one-dimensional distribution $\mathbb{P}\left(\mathbb{X}_{B}^{x}(t) \in d y\right)$ :

(a) Markov snake - when $|B(t)|$ increases we generate a new independent path. See Le Gall ([19], [20], and [21]) for applications to the nonlinear PDE $\Delta u=$ $u^{2}$.

(b) Let $X^{x, 1}(t), \ldots, X^{x, k}(t)$ be independent copies of $X^{x}(t)$ starting from point $x$. On each excursion interval of $|B(t)|$, use one of the $k$ copies chosen at random. When $x=0, X^{x}$ is a Brownian motion starting at 0 , and when $k=2$, this reduces to the iterated Brownian motion or IBM (see Burdzy [7, 8] Burdzy et al. 9, 10] and Khoshnevisan et al. [17]). We identify such a process by the abbreviation $k \mathrm{EBTP}$ and we denote it by $\mathbb{X}_{B, e}^{x, k}(t)$. Of course, when $k=1$ we obtain a BTP.

(c) Use an independent copy of $X^{x}$ on each excursion interval of $|B(t)|$. This is the $k \rightarrow \infty$ weak limit of (b) (for a rigorous statement and proof, see the Appendix of [1]). It is intermediate between IBM and the Markov snake. Here, we go forward on a new independent path only after $|B(t)|$ reaches 0 . This process is abbreviated as EBTP and is denoted by $\mathbb{X}_{B, e}^{x}(t)$.

As in the case of standard Brownian motion (and more generally diffusions), there is a host of interesting connections of BTPs to PDEs. However, unlike the Brownian motion's link to PDEs (see, e.g., [5, 6, 11, 16]), the PDEs here are fourth order and they are distinguished by the feature that the initial function is a fundamental part of the PDE itself, not only as initial data. We call such PDEs initially perturbed.

In this paper we always assume that the generator $\mathcal{A}$ of the outer Markov process $X^{x}$, and its associated semigroup $\mathcal{T}_{s}$, satisfy the property

$$
\begin{aligned}
& f: \mathbb{R}^{d} \rightarrow \mathbb{R} \text { bounded and } D_{i j} f \text { is Hölder continuous } \forall 1 \leq i, j \leq d \\
& \Longrightarrow \frac{\partial^{2} \mathcal{T}_{s} f(x)}{\partial s^{2}}=\mathcal{A}^{2} \mathcal{T}_{s} f \text { is continuous on }(0, \infty) \times \mathbb{R}^{d}, \text { and } \\
& \left\{\begin{array}{l}
(a) \mathcal{A}^{2} \int_{0}^{\infty} \mathcal{T}_{s} f(x) p_{t}(0, s) d s=\int_{0}^{\infty} \mathcal{A}^{2} \mathcal{T}_{s} f(x) p_{t}(0, s) d s ; \\
(b) \mathcal{A}^{2} \int_{0}^{t} \int_{0}^{\infty} \mathcal{T}_{s} f(x) p_{r}(0, s) d s d r=\int_{0}^{t} \int_{0}^{\infty} \mathcal{A}^{2} \mathcal{T}_{s} f(x) p_{r}(0, s) d s d r
\end{array}\right.
\end{aligned}
$$

where $p_{t}(0, s)$ is the transition density of the Brownian motion $B(t)$ and $D_{i j} f$ is $\partial^{2} / \partial x_{i} \partial x_{j}$. Property $(\mathbb{P})$ is satisfied when $X^{x}$ is a Brownian motion (see Lemma 2.1] below).

The first theorem gives us the fourth-order PDE solved by running a Browniantime process and then averaging the sum of $f\left(\mathbb{X}_{B}^{x}(t)\right)$ and the integral of a function $g$ along the path of $\mathbb{X}_{B}^{x}(t)$.

Theorem 1.1. Let $\mathcal{T}_{s} f(x)=\mathbb{E}_{\mathbb{P}} f\left(X^{x}(s)\right)$ be the semigroup of the continuous Markov process $X^{x}(t)$ and let $\mathcal{A}$ be its generator. Let $f$ and $g$ be bounded continuous functions in $\mathbb{D}(\mathcal{A})$, the domain of $\mathcal{A}$, such that $D_{i j} f$ and $D_{i j} g$ are bounded and Hölder continuous with exponent $0<\alpha \leq 1$, for all $1 \leq i, j \leq d$. If

$$
u(t, x)=\mathbb{E}_{\mathbb{P}}\left[f\left(\mathbb{X}_{B, e}^{x, k}(t)\right)+\int_{0}^{t} g\left(\mathbb{X}_{B, e}^{x, k}(r)\right) d r\right]
$$


for any $k \in \mathbb{N}$ (as stated before $\mathbb{X}_{B, e}^{x, 1}(t)=\mathbb{X}_{B}^{x}(t)$ ), or if we replace $\mathbb{X}_{B, e}^{x, k}(t)$ with $\mathbb{X}_{B, e}^{x}(t)$ in (1.1), then $u$ solves the PDE

$$
\begin{cases}\frac{\partial}{\partial t} u(t, x)=\frac{\mathcal{A} f(x)}{\sqrt{2 \pi t}}+\frac{\sqrt{2 t}}{\pi} \mathcal{A} g(x)+\frac{1}{2} \mathcal{A}^{2} u(t, x) ; & t>0, x \in \mathbb{R}^{d}, \\ u(0, x)=f(x)=\lim _{\substack{t \downarrow 0 \\ y \rightarrow x}} u(t, y) ; & x \in \mathbb{R}^{d},\end{cases}
$$

where the operator $\mathcal{A}$ acts on $u(t, x)$ as a function of $x$ with $t$ fixed. In particular, if $\mathbb{X}_{B}^{x}(t)$ is a BTBM and $\Delta$ is the standard Laplacian, then $u$ solves

$$
\begin{cases}\frac{\partial}{\partial t} u(t, x)=\frac{\Delta f(x)}{\sqrt{8 \pi t}}+\frac{\sqrt{2 t}}{2 \pi} \Delta g(x)+\frac{1}{8} \Delta^{2} u(t, x) ; & t>0, x \in \mathbb{R}^{d}, \\ u(0, x)=f(x)=\lim _{\substack{t \downarrow 0 \\ y \rightarrow x}} u(t, y) ; & x \in \mathbb{R}^{d} .\end{cases}
$$

Remark 1.1. The inclusion of the initial function $f(x)$ in the PDEs (1.2) and (1.3) is a reflection of the non-Markovian property of our BTP. Thus, as mentioned above, the role of $f$ here is fundamentally different from its role in the standard Markov-PDE connection. Moreover, as $t$ gets large, we see that the effect of the initial function $f$, through $\mathcal{A} f$, fades away at the rate $1 / \sqrt{2 \pi t}$, while the effect of $g$, through $\mathcal{A} g$, becomes more dominant at a rate $\sqrt{2 t} / \pi$. We also remark that property $(\mathbb{P})$ (excluding part $(b)$ ) should have been explicitly assumed for the case of the general outer Markov process $X^{x}$ in Theorem 0.1 in [1].

Next, we solve the PDE obtained by running an $\epsilon$-scaled BTP and averaging the product of $f\left(\mathbb{X}_{\epsilon B}^{x}(t)\right)$ with the negative exponential of $|B(t)| / \epsilon$ (the Brownian clock speeded up by $1 / \epsilon$ ). When $\epsilon=1$ this is a special case of the Feynman-Kac formula for BTP given by (1.8) in Theorem 1.3 However, it deserves to be singled out, for it is a first step towards the probabilistic study of linearized Cahn-Hilliard and Kuramoto-Sivashinsky type PDEs, which we undertake in an upcoming paper [3] (see also Remark 1.2 below).

Theorem 1.2. Under the same conditions on $f$ as in Theorem 1.1, and for $\epsilon>0$, if

$$
u_{\epsilon}(t, x) \triangleq \mathbb{E}_{\mathbb{P}}\left[f\left(\mathbb{X}_{\epsilon B, e}^{x, k}(t)\right) \exp \left(-\frac{|B(t)|}{\epsilon}\right)\right]
$$

for any $k \in \mathbb{N}$, or if we replace $\mathbb{X}_{\epsilon B, e}^{x, k}(t)$ with $\mathbb{X}_{\epsilon B, e}^{x}(t)$ in (1.4), then u solves

$$
\left\{\begin{aligned}
\frac{\partial}{\partial t} u_{\epsilon}(t, x)= & \frac{1}{\sqrt{2 \pi t}}\left[\epsilon \mathcal{A} f(x)-\frac{1}{\epsilon} f(x)\right] & & \\
& +\frac{1}{2 \epsilon^{2}} u_{\epsilon}(t, x)-\mathcal{A} u_{\epsilon}(t, x)+\frac{\epsilon^{2}}{2} \mathcal{A}^{2} u_{\epsilon}(t, x) ; & & t>0, x \in \mathbb{R}^{d}, \\
u_{\epsilon}(0, x)= & f(x)=\lim _{\substack{t \downarrow 0 \\
y \rightarrow x}} u_{\epsilon}(t, y) ; & & x \in \mathbb{R}^{d} .
\end{aligned}\right.
$$


In particular, if the outer Markov process $X^{x}$ in (1.4) is a Brownian motion, then $u_{\epsilon}(t, x)$ solves

$$
\left\{\begin{aligned}
\frac{\partial}{\partial t} u_{\epsilon}(t, x)= & \frac{1}{\sqrt{2 \pi t}}\left[\frac{\epsilon}{2} \Delta f(x)-\frac{1}{\epsilon} f(x)\right] & & \\
& +\frac{1}{2 \epsilon^{2}} u_{\epsilon}(t, x)-\frac{1}{2} \Delta u_{\epsilon}(t, x)+\frac{\epsilon^{2}}{8} \Delta^{2} u_{\epsilon}(t, x) ; & & t>0, x \in \mathbb{R}^{d}, \\
u_{\epsilon}(0, x)= & f(x)=\lim _{\substack{t \downarrow 0 \\
y \rightarrow x}} u_{\epsilon}(t, y) ; & & x \in \mathbb{R}^{d} .
\end{aligned}\right.
$$

Remark 1.2. This time the initial function $f$ affects our PDE through both $f$ and $\mathcal{A} f$; and, as before, these effects diminish as $t$ grows larger at the rate $1 / \sqrt{2 \pi t}$. Also, for small $\epsilon$, we see that the effects $f$ and $u_{\epsilon}$ are larger and eventually, as $\epsilon \searrow 0, u_{\epsilon}$ dominates all other terms in the PDE. We also comment briefly that, although a certainly different PDE, the last two terms in (1.6) (the bi-Laplacian and the Laplacian of the solution $u_{\epsilon}$ ) look like those in a linearized Cahn-Hilliard equation with the correct $\epsilon$-scaling, albeit with the opposite sign for $\Delta^{2}$.

The next result gives a Feynman-Kac type formula for BTPs and connects it to fourth-order PDEs:

Theorem 1.3. Assume that $f, c: \mathbb{R}^{d} \rightarrow \mathbb{R}$ are bounded, $c \leq 0$, and $D_{i j} f$ and $D_{i j} c$ are bounded and Hölder continuous with exponent $0<\alpha \leq 1$, for all $1 \leq i, j \leq d$. If the $\left|D_{i j} v(s, x)\right| \leq K_{T} \forall(s, x) \in[0, T] \times \mathbb{R}^{d}$, for any time $T>0$, for all $i, j$, where $K_{T}>0$ is a constant depending only on $T$ and

$$
v(s, x) \triangleq \mathbb{E}_{\mathbb{P}}\left[f\left(X^{x}(s)\right) \exp \left(\int_{0}^{s} c\left(X^{x}(r)\right) d r\right)\right],
$$

and where $X^{x}$ is a d-dimensional Brownian motion starting at $x$ under $\mathbb{P}$, then

$$
u(t, x) \triangleq \mathbb{E}_{\mathbb{P}}\left[f\left(\mathbb{X}_{B}^{x}(t)\right) \exp \left(\int_{0}^{|B(t)|} c\left(X^{x}(r)\right) d r\right)\right]
$$

solves

$$
\left\{\begin{aligned}
\frac{\partial}{\partial t} u(t, x)= & \frac{1}{\sqrt{2 \pi t}}\left[\frac{1}{2} \Delta f(x)+c(x) f(x)\right] & & \\
& +\left[\frac{1}{4} \Delta c(x)+\frac{1}{2} c^{2}(x)\right] u(t, x)+\frac{1}{2} \nabla c(x) \cdot \nabla u(t, x) & & \\
& +\frac{1}{2} c(x) \Delta u(t, x)+\frac{1}{8} \Delta^{2} u(t, x) ; & & t>0, x \in \mathbb{R}^{d}, \\
u_{\epsilon}(0, x)= & f(x)=\lim _{\substack{t \downarrow 0 \\
y \rightarrow x}} u_{\epsilon}(t, y) ; & & x \in \mathbb{R}^{d} .
\end{aligned}\right.
$$

Remark 1.3. As with previous PDEs, the effect of the initial function $f$ - this time through $\frac{1}{2} \Delta f(x)+c(x) f(x)$-fades away as $t$ grows larger at the BTP rate of $1 / \sqrt{2 \pi t}$. Another feature of the BTP Feynman-Kac PDE that is quite different from the standard Feynman-Kac PDE for Brownian motion is the existence of the interaction term $c(x) f(x)$ between the initial function $f$ and the function $c$. Also, we suspect that the conditions on $f$ and $c$ in Theorem 1.3 above are sufficient to imply the condition $\left|D_{i j} v(s, x)\right| \leq K_{T} \forall(s, x) \in[0, T] \times \mathbb{R}^{d}$, for any time $T>0$, for 
all $i, j$, but we do not have a proof of this yet. Finally, it is worth emphasizing that the BTP solutions to the PDEs presented in this article are all bounded.

\section{Proofs of Results}

2.1. A technical lemma. We start with a differentiating-under-the-integral type lemma.

Lemma 2.1. Let $X^{x}$ be a d-dimensional Brownian motion starting at $x$ under $\mathbb{P}$, and let $f, g: \mathbb{R}^{d} \rightarrow \mathbb{R}$ be bounded and measurable such that $D_{i j} f$ and $D_{i j} g$ are Hölder continuous, with exponent $0<\alpha \leq 1$, for $1 \leq i, j \leq d$. Let

$$
\begin{aligned}
& u_{1}(t, x) \triangleq \int_{0}^{\infty} \mathbb{E}_{\mathbb{P}} f\left(X^{x}(s)\right) p_{t}(0, s) d s, \\
& u_{2}(t, x) \triangleq \int_{0}^{t} \int_{0}^{\infty} \mathbb{E}_{\mathbb{P}} g\left(X^{x}(s)\right) p_{r}(0, s) d s d r .
\end{aligned}
$$

Then $\Delta^{2} u_{1}(t, x)$ and $\Delta^{2} u_{2}(t, x)$ are finite and

$$
\begin{aligned}
& \Delta^{2} u_{1}(t, x)=\int_{0}^{\infty} \Delta^{2} \mathbb{E}_{\mathbb{P}} f\left(X^{x}(s)\right) p_{t}(0, s) d s, \\
& \Delta^{2} u_{2}(t, x)=\int_{0}^{t} \int_{0}^{\infty} \Delta^{2} \mathbb{E}_{\mathbb{P}} g\left(X^{x}(s)\right) p_{r}(0, s) d s d r .
\end{aligned}
$$

If we additionally assume that the $D_{i j} f$ and $D_{i j} g$ are bounded (for all $i, j$ ), then $\Delta^{2} u_{1}(t, x)$ and $\Delta^{2} u_{2}(t, x)$ are continuous on $(0, \infty) \times \mathbb{R}^{d}$.

Proof. For notational simplicity we show that

$$
\begin{cases}\text { (a) } \frac{\partial^{4} u_{1}}{\partial x_{i}^{4}}=\int_{0}^{\infty} \frac{\partial^{4}}{\partial x_{i}^{4}} \mathbb{E}_{\mathbb{P}} f\left(X^{x}(s)\right) p_{t}(0, s) d s ; & i=1, \ldots, d, \\ (b) \frac{\partial^{4} u_{2}}{\partial x_{i}^{4}}=\int_{0}^{t} \int_{0}^{\infty} \frac{\partial^{4}}{\partial x_{i}^{4}} \mathbb{E}_{\mathbb{P}} g\left(X^{x}(s)\right) p_{r}(0, s) d s d r ; & i=1, \ldots, d,\end{cases}
$$

where the mixed derivatives cases follow the same steps. In the remainder of the proof, fix an arbitrary $i \in\{1, \ldots d\}$. We start with assertion (a) in (2.3). Using the boundedness on $f$ and Problem 3.1, p. 254 in [16] (the case $\mathbb{R}^{d}$ with $d>1$ is a simple extension when $f$ is bounded), the symmetry of $p_{s}^{(d)}(x, y)$ (the density of $\left.X^{x}\right)$ in $x$ and $y$, and the facts that

$$
\lim _{y_{i} \rightarrow \pm \infty} f(y) \frac{\partial^{3}}{\partial y_{i}^{3}} p_{s}^{(d)}(x, y)=\lim _{y_{i} \rightarrow \pm \infty} \frac{\partial}{\partial y_{i}} f(y) \frac{\partial^{2}}{\partial y_{i}^{2}} p_{s}^{(d)}(x, y)=0
$$

(since $f$ is bounded and $\frac{\partial}{\partial y_{i}} f(y)$ is Lipschitz in $y_{i}$ ), we get

$$
\begin{aligned}
& \frac{\partial^{4}}{\partial x_{i}^{4}} \mathbb{E}_{\mathbb{P}} f\left(X^{x}(s)\right) p_{t}(0, s)=\left(\int_{\mathbb{R}^{d}} f(y) \frac{\partial^{4}}{\partial x_{i}^{4}} p_{s}^{(d)}(x, y) d y\right) p_{t}(0, s) \\
& =\left(\int_{\mathbb{R}^{d}} f(y) \frac{\partial^{4}}{\partial y_{i}^{4}} p_{s}^{(d)}(x, y) d y\right) p_{t}(0, s) \\
& =\left(\int_{\mathbb{R}^{d}} \frac{\partial^{2}}{\partial y_{i}^{2}} f(y) \frac{\partial^{2}}{\partial y_{i}^{2}} p_{s}^{(d)}(x, y) d y\right) p_{t}(0, s) .
\end{aligned}
$$


Rewriting the last term in (2.4), and letting $h_{i}(y) \triangleq \partial^{2} f(y) / \partial y_{i}^{2}$, we have

$$
\begin{aligned}
& \frac{1}{\sqrt{2 \pi t}}\left(\int_{\mathbb{R}^{d}}(2 \pi s)^{-d / 2}\left(\frac{\left(x_{i}-y_{i}\right)^{2}-s}{s^{2}}\right) e^{-|x-y|^{2} / 2 s} h_{i}(y) d y\right) e^{-s^{2} / 2 t} \\
& =\frac{1}{\sqrt{2 \pi t}} e^{-s^{2} / 2 t} \mathbb{E}_{\mathbb{P}}\left[\left(\frac{\left(x_{i}-X_{i}^{x}(s)\right)^{2}-s}{s^{2}}\right) h_{i}\left(X^{x}(s)\right)\right] \\
& =\frac{1}{\sqrt{2 \pi t}} e^{-s^{2} / 2 t} \mathbb{E}_{\mathbb{P}}\left[\left(\frac{\left(x_{i}-X_{i}^{x}(s)\right)^{2}-s}{s^{2}}\right)\left(h_{i}\left(X^{x}(s)\right)-h_{i}(x)\right)\right],
\end{aligned}
$$

where we used the fact that $\mathbb{E}_{\mathbb{P}}\left(\left(x_{i}-X_{i}^{x}(s)\right)^{2}-s\right)=0$ to obtain the last equality. Now, using the Brownian motion scaling, we have

$$
\mathbb{E}_{\mathbb{P}}\left|\left(x_{i}-X_{i}^{x}(s)\right)^{2}-s\right|^{2}=s^{2} \mathbb{E}_{\mathbb{P}}\left|\left(\frac{X_{i}^{0}(s)}{\sqrt{s}}\right)^{2}-1\right|^{2}=s^{2} \mathbb{E}_{\mathbb{P}}\left|\left(X_{i}^{0}(1)\right)^{2}-1\right|^{2}=C s^{2},
$$

for some constant $C$; so an easy application of the Cauchy-Schwarz inequality yields

$$
\begin{aligned}
& \left|\frac{\partial^{4}}{\partial x_{i}^{4}} \mathbb{E}_{\mathbb{P}} f\left(X^{x}(s)\right) p_{t}(0, s)\right| \\
& \leq \frac{1}{\sqrt{2 \pi t}} e^{-s^{2} / 2 t}\left(\mathbb{E}_{\mathbb{P}}\left|\frac{\left(x_{i}-X_{i}^{x}(s)\right)^{2}-s}{s^{2}}\right|^{2} \mathbb{E}_{\mathbb{P}}\left|h_{i}\left(X^{x}(s)\right)-h_{i}(x)\right|^{2}\right)^{1 / 2} \\
& \leq \frac{K}{\sqrt{2 \pi t}} \frac{e^{-s^{2} / 2 t}}{s}\left(\mathbb{E}_{\mathbb{P}}\left|X^{x}(s)-x\right|^{2 \alpha}\right)^{1 / 2}=\frac{K}{\sqrt{2 \pi t}} \frac{e^{-s^{2} / 2 t}}{s^{1-\alpha / 2}},
\end{aligned}
$$

where the next to last inequality follows from (2.6) and the Hölder condition on $h_{i}$. But

$$
\frac{K}{\sqrt{2 \pi t}} \int_{0}^{\infty} \frac{e^{-s^{2} / 2 t}}{s^{1-\alpha / 2}} d s<\infty,
$$

for $\alpha>0$. So, 2.3 (a), as well as the continuity assertion afterwards, follow by a standard classical argument (see, e.g., Friedman [13, pages 10-12 for the purely analytical details in the second-order case).

Now, by part (a) of (2.3), part (b) of (2.3) is established once we show

$$
\frac{\partial^{4}}{\partial x_{i}^{4}} \int_{0}^{t} \int_{0}^{\infty} \mathbb{E}_{\mathbb{P}} g\left(X^{x}(s)\right) p_{r}(0, s) d s d r=\int_{0}^{t} \frac{\partial^{4}}{\partial x_{i}^{4}} \int_{0}^{\infty} \mathbb{E}_{\mathbb{P}} g\left(X^{x}(s)\right) p_{r}(0, s) d s d r .
$$

This is simple, however, since by the first part we have

$$
\begin{aligned}
\left|\frac{\partial^{4}}{\partial x_{i}^{4}} \int_{0}^{\infty} \mathbb{E}_{\mathbb{P}} g\left(X^{x}(s)\right) p_{r}(0, s) d s\right| & =\left|\int_{0}^{\infty} \frac{\partial^{4}}{\partial x_{i}^{4}} \mathbb{E}_{\mathbb{P}} g\left(X^{x}(s)\right) p_{r}(0, s) d s\right| \\
& \leq \int_{0}^{\infty}\left|\frac{\partial^{4}}{\partial x_{i}^{4}} \mathbb{E}_{\mathbb{P}} g\left(X^{x}(s)\right) p_{r}(0, s)\right| d s \\
& \leq \frac{K}{\sqrt{2 \pi r}} \int_{0}^{\infty} \frac{e^{-s^{2} / 2 r}}{s^{1-\alpha / 2}} d s
\end{aligned}
$$

and

$$
\int_{0}^{t}\left(\frac{K}{\sqrt{2 \pi r}} \int_{0}^{\infty} \frac{e^{-s^{2} / 2 r}}{s^{1-\alpha / 2}} d s\right) d r<\infty
$$


So, again standard arguments (e.g., Problem 3, p. 52 in 44 or Friedman again) complete the proof.

Remark 2.1. By an identical argument to that in Lemma 2.1 above, with only notational differences to accommodate the dependence on time $r$ in $g(r, x), \Delta^{2}$ can be pulled outside the integrals in (2.25) once $D_{i j} g$ is continuous on $[0, T] \times \mathbb{R}^{d}$ and Hölder continuous in $x$ uniformly with respect to $(t, x) \in[0, T] \times \mathbb{R}^{d}$.

2.2. The main proofs. In all the proofs presented here, it suffices to prove the result for the BTP case. The excursion BTP (including the IBM) cases are proved from the BTP one in exactly the same way as in the proof of Theorem 0.1 in [1].

Proof of Theorem [1.1. We first use the independence of $X^{x}(\cdot)$ and $|B(\cdot)|$ to get

$$
\begin{aligned}
u(t, x) & =\mathbb{E}_{\mathbb{P}}\left[f\left(\mathbb{X}_{B}^{x}(t)\right)+\int_{0}^{t} g\left(\mathbb{X}_{B}^{x}(r)\right) d r\right] \\
& =2 \int_{0}^{\infty} \mathcal{T}_{s} f(x) p_{t}(0, s) d s+2 \int_{0}^{\infty} \int_{0}^{t} \mathcal{T}_{s} g(x) p_{r}(0, s) d r d s
\end{aligned}
$$

where $p_{t}(0, s)$ is the transition density of $B(t)$, and where we used the boundedness of $g$ and consequently that of $\mathcal{T}_{s} g(x)$ along with Fubini's theorem to get the last term. Differentiating (2.11) with respect to $t$ and putting the derivative under the integral, which is easily justified by the dominated convergence theorem (remember that $f$ and $g$ are bounded), then using the fact that $p_{t}(0, s)$ satisfies the heat equation

$$
\frac{\partial}{\partial t} p_{t}(0, s)=\frac{1}{2} \frac{\partial^{2}}{\partial s^{2}} p_{t}(0, s)
$$

we have

$$
\begin{aligned}
\frac{\partial}{\partial t} u(t, x) & =2 \int_{0}^{\infty} \mathcal{T}_{s} f(x) \frac{\partial}{\partial t} p_{t}(0, s) d s+2 \int_{0}^{\infty} \mathcal{T}_{s} g(x)\left(\int_{0}^{t} \frac{\partial}{\partial r} p_{r}(0, s) d r\right) d s \\
& =\int_{0}^{\infty} \mathcal{T}_{s} f(x) \frac{\partial^{2}}{\partial s^{2}} p_{t}(0, s) d s+\int_{0}^{t} \int_{0}^{\infty} \mathcal{T}_{s} g(x) \frac{\partial^{2}}{\partial s^{2}} p_{r}(0, s) d s d r .
\end{aligned}
$$

So, integrating by parts twice and observing that the boundary terms always vanish at $\infty($ as $s \nearrow \infty)$ and that $\left.(\partial / \partial s) p_{t}(0, s)\right|_{s=0}=0$ but $p_{t}(0,0)>0$, we get

$$
\begin{aligned}
\frac{\partial}{\partial t} u(t, x) & =\left.p_{t}(0,0)\left(\frac{\partial}{\partial s} \mathcal{T}_{s} f(x)\right)\right|_{s=0}+\left.\int_{0}^{t} p_{r}(0,0)\left(\frac{\partial}{\partial s} \mathcal{T}_{s} g(x)\right)\right|_{s=0} d r \\
& +\int_{0}^{\infty} \mathcal{A}^{2} \mathcal{T}_{s} f(x) p_{t}(0, s) d s+\int_{0}^{t} \int_{0}^{\infty} \mathcal{A}^{2} \mathcal{T}_{s} g(x) p_{r}(0, s) d s d r \\
& =\frac{1}{\sqrt{2 \pi t}} \mathcal{A} f(x)+\frac{\sqrt{2 t}}{\pi} \mathcal{A} g(x) \\
& +\mathcal{A}^{2}\left(\int_{0}^{\infty} \mathcal{T}_{s} f(x) p_{t}(0, s) d s+\int_{0}^{t} \int_{0}^{\infty} \mathcal{T}_{s} g(x) p_{r}(0, s) d s d r\right) \\
& =\frac{1}{\sqrt{2 \pi t}} \mathcal{A} f(x)+\frac{\sqrt{2 t}}{\pi} \mathcal{A} g(x)+\frac{1}{2} \mathcal{A}^{2} u(t, x),
\end{aligned}
$$


where in the next to last step we used property $(\mathrm{P})$. Obviously, $u(0, x)=f(x)$. Also, rewriting $u$ as $u(t, x)=\mathbb{E}_{\mathbb{P}} f\left(x+\mathbb{X}_{B}^{0}(t)\right)$ and noticing that $f$ is bounded and continuous, we can use the bounded convergence theorem to conclude

$$
f(x)=\lim _{\substack{t \downarrow 0 \\ y \rightarrow x}} u(t, y) .
$$

Next, we present the

Proof of Theorem 1.2. Again, it is enough to prove the BTP case. Let

$$
u_{\epsilon}(t, x) \triangleq \mathbb{E}_{\mathbb{P}}\left[f\left(\mathbb{X}_{\epsilon B}^{x}(t)\right) \exp \left(-\frac{|B(t)|}{\epsilon}\right)\right]
$$

and

$$
v_{\epsilon}(s, x) \triangleq \mathbb{E}_{\mathbb{P}}\left[f\left(X^{x}(\epsilon s)\right) \exp \left(-\frac{s}{\epsilon}\right)\right]=\exp \left(-\frac{s}{\epsilon}\right) \mathcal{T}_{\epsilon s} f(x) .
$$

We then have

$$
u_{\epsilon}(t, x)=2 \int_{0}^{\infty} v_{\epsilon}(s, x) p_{t}(0, s) d s,
$$

and so, following our argument in the previous proof, and noticing that, for a fixed $\epsilon$, $\exp (-s / \epsilon)$ and all of its derivatives are bounded and in $C^{\infty}\left(\mathbb{R}_{+} ; \mathbb{R}_{+}\right)$, we get

$$
\begin{aligned}
\frac{\partial}{\partial t} u_{\epsilon}(t, x) & =2 \int_{0}^{\infty} v_{\epsilon}(s, x) \frac{\partial}{\partial t} p_{t}(0, s) d s=\int_{0}^{\infty} v_{\epsilon}(s, x) \frac{\partial^{2}}{\partial s^{2}} p_{t}(0, s) d s \\
& =\left.p_{t}(0,0)\left(\frac{\partial}{\partial s} v_{\epsilon}(s, x)\right)\right|_{s=0}+\int_{0}^{\infty} p_{t}(0, s) \frac{\partial^{2}}{\partial s^{2}} v_{\epsilon}(s, x) d s \\
& =\frac{1}{\sqrt{2 \pi t}}\left[\epsilon \mathcal{A} f(x)-\frac{1}{\epsilon} f(x)\right]+\frac{1}{2 \epsilon^{2}} u_{\epsilon}(t, x)-\mathcal{A} u_{\epsilon}(t, x)+\frac{\epsilon^{2}}{2} \mathcal{A}^{2} u_{\epsilon}(t, x),
\end{aligned}
$$

where we have also used property $(\mathrm{P})$. Again, $u_{\epsilon}(0, x)=f(x)$. Rewriting $u$ as $u(t, x)=\mathbb{E}_{\mathbb{P}}\left[f\left(x+\mathbb{X}_{\epsilon B}^{0}(t)\right) \exp (-|B(t)| / \epsilon)\right]$ and noticing that $f$ is bounded and continuous, we can use the bounded convergence theorem to conclude

$$
f(x)=\lim _{\substack{t \downarrow 0 \\ y \rightarrow x}} u_{\epsilon}(t, y) .
$$

We are now in a position to give the

Proof of Theorem 1.3. Let $u$ and $v$ be defined as in (1.8) and (1.7), respectively. Then, as we did several times above,

$$
u(t, x)=2 \int_{0}^{\infty} p_{t}(0, s) v(s, x) d s .
$$

Differentiating (2.17) with respect to $t$ and putting the derivative under the integral, which is again justified by the dominated convergence theorem (remember that $f$ is bounded and $c \leq 0$ ), then proceeding as in the proof of Theorem 1.1 and noting that, since $X^{x}$ is Brownian motion starting at $x$ under $\mathbb{P}$, then

$$
\frac{\partial}{\partial s} v(s, x)=\frac{1}{2} \Delta v(s, x)+c(x) v(s, x), \text { in }(0, \infty) \times \mathbb{R}^{d},
$$


with $v$ continuous on $[0, \infty) \times \mathbb{R}^{d}$ and $v(0, x)=f(x)$ (see, for example, section 4.3 of [11]). So, integrating by parts twice, and again observing that the boundary terms always vanish at $\infty($ as $s \nearrow \infty)$ and that $\left.(\partial / \partial s) p_{t}(0, s)\right|_{s=0}=0$ but $p_{t}(0,0)>0$, we get

$$
\begin{aligned}
\frac{\partial}{\partial t} u(t, x) & =-\int_{0}^{\infty} \frac{\partial}{\partial s} p_{t}(0, s) \frac{\partial}{\partial s} v(s, x) d s \\
& =\left.p_{t}(0,0)\left(\frac{\partial}{\partial s} v(s, x)\right)\right|_{s=0}+\int_{0}^{\infty} p_{t}(0, s) \frac{\partial^{2}}{\partial s^{2}} v(s, x) d s \\
& =p_{t}(0,0)\left(\frac{1}{2} \Delta f(x)+c(x) f(x)\right) \\
& +\int_{0}^{\infty} p_{t}(0, s)\left(\frac{1}{4} \Delta^{2} v(s, x)+\frac{1}{2} v(s, x) \Delta c(x)\right) d s \\
& +\int_{0}^{\infty} p_{t}(0, s)\left(\nabla c(x) \cdot \nabla v(s, x)+c(x) \Delta v(s, x)+c^{2}(x) v(s, x)\right) d s .
\end{aligned}
$$

Taking the application of $\Delta, \nabla, \Delta^{2}$ as well as the terms $c, c^{2}, \Delta c, \nabla c$ and the dot product outside the integral, we get the PDE in (1.2). To justify this last step, it suffices to show that we can take the highest-order derivatives $\left(\Delta^{2}\right)$ outside the integral. Towards this end, we first note that

$$
v(s, x)=\mathbb{E}_{\mathbb{P}} f\left(X^{x}(s)\right)+\int_{0}^{s} \mathbb{E}_{\mathbb{P}}\left\{c\left(X^{x}(r)\right) v\left(s-r, X^{x}(r)\right)\right\} d r .
$$

This follows from exactly the same steps as those in Durrett's [1], pp. 140-141. Then,

$$
D_{i j} v(s, x)=D_{i j} \mathbb{E}_{\mathbb{P}} f\left(X^{x}(s)\right)+D_{i j}\left(\int_{0}^{s} \mathbb{E}_{\mathbb{P}}\left\{g\left(s-r, X^{x}(r)\right)\right\} d r\right) ; 1 \leq i, j \leq d,
$$

where $g(r, x)=c(x) v(r, x)$. Fix an arbitrary pair $i, j$ and let

$$
v_{1}(s, x) \triangleq D_{i j} \mathbb{E}_{\mathbb{P}} f\left(X^{x}(s)\right) \text { and } v_{2}(s, x) \triangleq D_{i j} \int_{0}^{s} \mathbb{E}_{\mathbb{P}}\left\{g\left(s-r, X^{x}(r)\right)\right\} d r .
$$

We see from the boundedness of $f$ and the Hölder and boundedness assumptions on $D_{i j} f$ that

$$
\begin{aligned}
\left|v_{1}(s, x)-v_{1}(s, y)\right| & =\left|\mathbb{E}_{\mathbb{P}}\left[D_{i j} f\left(x+X^{0}(s)\right)-D_{i j} f\left(y+X^{0}(s)\right)\right]\right| \\
& \leq \mathbb{E}_{\mathbb{P}}\left|D_{i j} f\left(x+X^{0}(s)\right)-D_{i j} f\left(y+X^{0}(s)\right)\right| \leq C|x-y|^{\alpha} .
\end{aligned}
$$

In fact, the boundedness of $f$ and Problem 3.1 in [16] imply that $D_{i j} \mathbb{E}_{\mathbb{P}} f\left(X^{x}(s)\right)=$ $\mathbb{E}_{\mathbb{P}} D_{i j} f\left(x+X^{0}(s)\right)$ has derivatives of all orders (in both $s$ and $x$, for $(s, x) \in$ $\left.(0, \infty) \times \mathbb{R}^{d}\right)$, and hence is Lipschitz in $x$.

Now, the boundedness of $v$ (implied by the boundedness of $f$ and the fact that $c \leq 0)$ and the boundedness of $c$ imply the boundedness of $g$. This, in addition to Theorem 2.6c in [11] (in Chapter 4) yield

$$
v_{2}(s, x)=\int_{0}^{s} \int_{\mathbb{R}^{d}} g(s-r, y) D_{i j} p_{r}^{(d)}(x, y) d r d y .
$$


But $\left|D_{i j} v(s, x)\right| \leq K_{T} \forall(s, x) \in[0, T] \times \mathbb{R}^{d}$, for any time $T>0$, by assumption; and $D_{i j} v$ is continuous by Theorem 3.6, p. 140 in [11] (since $f$ and $c$ are both bounded by assumption and Hölder continuous because $D_{i j} f$ and $D_{i j} c$ are by assumption). This, in addition to the assumption that $D_{i j} c$ are all Hölder continuous and bounded imply that $D_{i j} g(s, x)$ is bounded on $[0, T]$ uniformly in $x$ and continuous, so that, if $G(r, y) \triangleq D_{i j} g(r, y)$, then (2.22) implies that

$$
v_{2}(s, x)=\int_{0}^{s} \int_{\mathbb{R}^{d}} p_{r}^{(d)}(x, y) D_{i j} g(s-r, y) d r d y=\int_{0}^{s} \int_{\mathbb{R}^{d}} p_{r}^{(d)}(x, y) G(s-r, y) d r d y,
$$

and it follows by Theorem 2.6b, pp. 133-134 in [11] (in which the assertion of differentiability and continuity of derivatives is unaffected if we replace $|G| \leq M$ with $\left.|G(t, x)| \leq M_{T} \forall(t, x) \in[0, T] \times \mathbb{R}^{d}, \forall T\right)$ that

$$
\left|v_{2}(s, x)-v_{2}(s, y)\right| \leq C_{T}|x-y|, \forall s \in[0, T] .
$$

Clearly, (2.21) and (2.24) imply that $D_{i j} v(s, x)=v_{1}(s, x)+v_{2}(s, x)$ is Hölder continuous with exponent $\alpha$ in $x$ uniformly with respect to $(s, x) \in[0, T] \times \mathbb{R}^{d}$. So, by the boundedness assumptions on $D_{i j} v$ and $D_{i j} c$ and the assumption that $D_{i j} c$ is Hölder continuous, it follows that $D_{i j} g(s, x)$ is Hölder continuous with exponent $\alpha$ in $x$ uniformly with respect to $(s, x) \in[0, T] \times \mathbb{R}^{d}$.

Now, since $f, g$ and $D_{i j} f, D_{i j} g$ are bounded and Hölder continuous, then (2.19), Lemma 2.1 and Remark 2.1 imply that

$$
\begin{aligned}
& \Delta^{2} \int_{0}^{\infty} p_{t}(0, s) v(s, x) d s \\
& =\Delta^{2} \int_{0}^{\infty} p_{t}(0, s)\left(\mathbb{E}_{\mathbb{P}} f\left(X^{x}(s)\right)+\int_{0}^{s} \mathbb{E}_{\mathbb{P}}\left\{g\left(s-r, X^{x}(r)\right)\right\} d r\right) d s \\
& =\Delta^{2} \int_{0}^{\infty} p_{t}(0, s) \mathbb{E}_{\mathbb{P}} f\left(X^{x}(s)\right) d s+\Delta^{2} \int_{0}^{\infty} p_{t}(0, s) \int_{0}^{s} \mathbb{E}_{\mathbb{P}}\left\{g\left(s-r, X^{x}(r)\right)\right\} d r d s \\
& =\int_{0}^{\infty} p_{t}(0, s) \Delta^{2} \mathbb{E}_{\mathbb{P}} f\left(X^{x}(s)\right) d s+\int_{0}^{\infty} p_{t}(0, s) \int_{0}^{s} \Delta^{2} \mathbb{E}_{\mathbb{P}}\left\{g\left(s-r, X^{x}(r)\right)\right\} d r d s \\
& =\int_{0}^{\infty} p_{t}(0, s)\left(\Delta^{2} \mathbb{E}_{\mathbb{P}} f\left(X^{x}(s)\right)+\Delta^{2} \int_{0}^{s} \mathbb{E}_{\mathbb{P}}\left\{g\left(s-r, X^{x}(r)\right)\right\} d r\right) d s \\
& =\int_{0}^{\infty} p_{t}(0, s) \Delta^{2} v(s, x) d s .
\end{aligned}
$$

So, we can pull the operator $\Delta^{2}$ outside the integral in (2.18) as desired.

Obviously, $u(0, x)=f(x)$, and rewriting $u$ as

$$
u(t, x)=\mathbb{E}_{\mathbb{P}}\left[f\left(x+\mathbb{X}_{B}^{0}(t)\right) \exp \left(\int_{0}^{|B(t)|} c\left(x+X^{0}(r)\right) d r\right)\right]
$$

and noticing that $f$ is bounded and continuous and $c \leq 0$, bounded and continuous, we can use the bounded convergence theorem to conclude

$$
f(x)=\lim _{\substack{t \downarrow 0 \\ y \rightarrow x}} u(t, y)
$$

so that $u(t, x)$ solves (1.9). 
Acknowledgement. I would like to thank the referee for his nice and constructive comments.

\section{REFERENCES}

[1] H. Allouba and W. Zheng, Brownian-time processes: the PDE connection and the halfderivative generator. Ann. of Probab. 29 no. 4, (2001), 1780-1795.

[2] H. Allouba, Measure-valued Brownian-time processes: the PDE connection. (2001). In preparation.

[3] H. Allouba, Brownian-time type processes: the PDE connection III. (2001). In preparation

[4] R. Ash, Real analysis and probability. Academic Press, Inc., New York, 1972. MR 55:8280

[5] R. Bass, Probabilistic techniques in analysis. Springer-Verlag, New York, $1995 . \quad$ MR 96e:60001

[6] R. Bass, Diffusions and elliptic operators. Springer-Verlag, New York, 1997. MR 99h:60136

[7] K. Burdzy, Some path properties of iterated Brownian motion. Seminar on Stochastic Processes 1992, Birkhäuser, (1993), 67-87. MR 95c:60075

[8] K. Burdzy, Variation of iterated Brownian motion. Workshop and conference on measurevalued processes, stochastic PDEs and interacting particle systems. CRM Proceedings and Lecture Notes 5, (1994), 35-53. MR 95h:60123

[9] K. Burdzy and D. Khoshnevisan, The level sets of iterated Brownian motion. Séminaire de Probabilités XXIX. Lecture Notes in Math. 1613, (1995), 231-236. MR 98k:60138

[10] K. Burdzy and D. Khoshnevisan, Brownian motion in a Brownian crack. Ann. Appl. Probab. 8 no. 3, (1998), 708-748. MR 99g:60147

[11] R. Durrett, Stochastic calculus, a practical introduction. Probability and Stochastics Series. CRC Press, Boca Raton, FL., 1996. MR 97k:60148

[12] K. D. Elworthy, Stochastic differential equations on manifolds, Cambridge University Press, 1982. MR 84d:58080

[13] A Friedman, Partial Differential Equations of Parabolic Type, Prentice-Hall, Englewood Cliffs, NJ, 1964. MR 31:6062

[14] T. Funaki, Probabilistic construction of the solution of some higher order parabolic differential equation. Proc. Japan Acad. Ser. A Math. Sci. 55 no. 5, (1979), 176-179. MR 80h:60075

[15] K. Hochberg and E. Orsingher, Composition of stochastic processes governed by higherorder parabolic and hyperbolic equations. J. Theoret. Probab. 9, no. 2, (1996), 511-532. MR 97f:60181

[16] I. Karatzas and S. Shreve, Brownian motion and stochastic calculus. Springer-Verlag, 1988. MR 89c:60096

[17] D. Khoshnevisan and T. Lewis Iterated Brownian motion and its intrinsic skeletal structure. Seminar on Stochastic Analysis, Random Fields and Applications (Ascona, 1996), 201-210, Progr. Probab., 45, Birkhäuser, Basel, 1999. MR 2001m:60183

[18] H. Kunita, Stochastic flows and stochastic differential equations, Cambridge University Press, 1990. MR 91m:60107

[19] J-F. Le Gall, Solutions positives de $\Delta u=u^{2}$ dans le disque unité. C. R. Acad. Sci. Paris Série I. 317, (1993), 873-878. MR 94h:35059

[20] J-F. Le Gall, A path-valued Markov process and its connections with partial differential equations. First European Congress of Mathematics, Vol. II (Paris, 1992). Progr. Math. 120, Birkhäuser, (1994), 185-212. MR 96m:60169

[21] J-F. Le Gall, The Brownian snake and solutions of $\Delta u=u^{2}$ in a domain. Probab. Theory Related Fields. 102 no. 3, (1995), 393-432. MR 96c:60098

[22] T. Lyons, and W. Zheng, On conditional diffusion processes. Proc. Roy. Soc. Edinburgh. 115, (1990), 243-255. MR 91m:60148

[23] W. Zheng, Conditional propagation of chaos and a class of quasilinear PDE's. Ann. Probab. 23 no. 3, (1995), 1389-1413. MR 96m:60183

Department of Mathematics, Indiana University, Bloomington, Indiana 47405-7106

$U R L:$ http://php.indiana.edu/ ${ }^{\sim}$ allouba

E-mail address: allouba@indiana.edu

Current address: Department of Mathematical Sciences, Kent State University, Kent, Ohio 44240-0001 